\title{
Inoculación in situ de Morchella: primer registro de su obtención en México
}

\author{
Morchella inoculation in situ: first record in México
}

\author{
Gerardo Alvarado-Castillo', Griselda Benítez-Badillo² y Gerardo Mata ${ }^{3 *}$
}

'Facultad de Ciencias Agrícolas. Posgrado en Ciencias Agropecuarias. Universidad Veracruzana. Xalapa, Veracruz, México.

\author{
${ }^{2}$ Red Ambiente y Sustentabilidad. Instituto de Ecolo- \\ gía A.C., Xalapa, Veracruz, México.
}

${ }^{3}$ Red Manejo Biotecnológico de Recursos. Instituto de Ecología A.C., Xalapa, Veracruz, México.

*Autor para correspondencia. gerardo.mata@inecol.mx

\section{RESUMEN}

Se documenta el primer caso de obtención de Morchella en México, por medio de su inoculación in situ. Este hongo es económicamente importante por su alta demanda en el mercado nacional e internacional, pero a pesar de que se han generado patentes para su producción comercial, su obtención aún depende básicamente de su recolección en campo, lo cual tiene repercusiones en el hábitat y ciclo de vida de la especie. Se realizó un experimento en laboratorio para evaluar el crecimiento micelial de dos especies de Morchella en cuatro tipos de granos (maíz, avena, trigo y centeno), para determinar cuál es el más apropiado como agente inoculante. Las cepas utilizadas fueron: M. esculenta (IE-750) y M. conica (IE-815, IE-817, IE-816 y IE-814), de las cuales el mejor tratamiento fue IE-817 en centeno, el cual posteriormente fue inoculado en el suelo de un predio ubicado en un bosque mesófilo de montaña perturbado (con predominancia de especies del género Inga, Liquidambar y Sambucus). Como resultado se consiguieron tres periodos de fructificación entre el año 2009 y 2011. Esto indica la posibilidad de obtención de este hongo, a través de estrategias de producción in situ, lo cual impulsaría un nuevo enfoque para su producción bajo un esquema de micosilvicultura.

Palabras Clave: cultivo in situ, esclerocios, inoculación, micosilvicultura.

\section{ABSTRACT}

The first case of obtaining Morchella in Mexico by inoculation in situ is presented. This mushroom is economically important for its high demand in the domestic and international market, but despite the fact that some patents for commercial production have been generated; their production still depends essentially on field harvesting. An experiment was conducted in the laboratory to evaluate the mycelial growth of two species of Morchella in four types of grains (corn, oats, wheat and rye), to determine which is the most appropriate inoculant agent. The strains used were: M. esculenta (IE-750) and M. conica (IE-815, 817-IE, IE-816 and IE-814), of which the best treatment was IE-817 in rye, which was subsequently injected into the soil of a piece of land located in a cloud forest mountain disturbed (with predominance of species of the genus Inga, Liquidambar, and Sambucus). As a result three fruiting periods were obtained between in 2009 and 2011. This indicates the possibility of obtaining this mushroom through production strategies in situ, which would trigger a new approach to production under a mycosilviculture scheme.

KEYWORDs: in situ cultivation, sclerotia, inoculation, mycosilviculture.

\section{INTRODUCCIÓN}

El aprovechamiento forestal usualmente se ha centrado en la producción de madera, sin embargo, los altos costos de producción, la globalización (competencia con las importaciones) y las preocupaciones públicas para la protección del ecosistema (Martínez de Aragón et al., 2011) hacen necesario un nuevo enfoque de manejo del bosque. Este esquema debe ser multifuncional y sostenible de tal manera que integre funciones productivas, ecológicas y sociales. En este sentido la micosilvicultura, busca inte- 
grar la producción de madera y hongos, aprovechando la sinergia entre estos dos elementos (Bonet et al., 2010).

Este esquema productivo se basa en que gran parte de los hongos silvestres comestibles, mantienen una relación benéfica con los arboles del bosque, contribuyendo a su nutrición (mineralización, inmovilización, óxido-reducción, volatilización, fijación, precipitación y solubilización de nutrientes), favorecen el equilibrio de los ciclos biogeoquímicos (Savoie y Largeteau, 2011; Martínez-Peña et al., 2012), forman parte de la cadena trófica y colaboran a la salud del sistema forestal (Montoya y Orrego, 2012). A su vez la productividad de los hongos depende del sistema forestal y del ambiente ecológico (comunidad vegetal, clima, suelo, desarrollo de árbol, etc.), de tal manera que el manejo forestal puede favorecer la obtención de ambos productos y contribuir a la resiliencia de los bosques (Savoie y Largeteau, 2011).

Adicionalmente, la recolección de hongos ha demostrado una rentabilidad igual o mayor (hasta $60 \%$ más) que la producción maderable (Bonet et al., 2010, 2012; Martínez de Aragón et al., 2011) y la creciente demanda de estos productos forestales no maderables (PFNM) por sus propiedades nutracéuticas, han aumentado la importancia relativa de los hongos en el bosque. No obstante, en algunos casos, la falta de orientación técnica y científica en el aprovechamiento, aumenta la probabilidad de sobreexplotación o extinción de las especies tradicionales más conocidas, tal es el caso de los ascomicetos del género Morchella.

Estos hongos tienen un alto valor comercial a nivel nacional e internacional (Masaphy, 2005), por lo cual se han realizado numerosos intentos para cultivarlos artificialmente. A pesar de la generación de dos patentes (Ower, 1982; Ower et al., 1986, 1988) aún existen dificultades para producirlo de forma masiva (Masaphy, 2005) pues en la práctica hay una serie de limitantes que no han podido superarse, tales como la nula y baja productividad, aborto de primordios y malformación de ascocarpos (Molina et al., 1993; Barnes y Wilson, 1998; Stott y Mohammed, 2004).

A la fecha, el conocimiento generado no permite el cultivo de este hongo bajo condiciones controladas, por lo que su aprovechamiento se restringe a la recolección de sus poblaciones naturales, la cual generalmente es realizada de forma desigual y desordenada, provocando impactos tales como la perturbación excesiva del hábitat, compactación del suelo (Martínez de Aragón et al., 2011), interrupción del ciclo biológico de algunas especies, fluctuación y disminución de su abundancia y erosión genética (Tacón y Palma, 2006; Pilz et al., 2007), que no solo interfieren en el desarrollo de este hongo, sino además en el importante papel que juegan en la salud forestal (Bonet et al., 2012; Montoya y Orrego, 2012).

La escasez, estacionalidad y los altos precios que alcanzan estos hongos, generan una alta presión extractiva sobre sus poblaciones naturales, por lo que las seis especies que existen en México se encuentran catalogadas como amenazadas en la norma de protección NOM059-Semarnat-2010 (Semarnat, 2010). Ante este panorama, las opciones posibles hoy en día para el aprovechamiento racional de este y otros hongos silvestres comestibles son: el manejo y gestión de las poblaciones naturales bajo un esquema de micosilvicultura y el desarrollo de técnicas para su domesticación y producción comercial.

En ambos casos, las prácticas de micorrización controlada, la inoculación in situ y prácticas de manejo forestal (raleo, aclareo y podas), pueden ofrecer un avance prometedor para la obtención de estos y otros hongos (Bonet et al., 2012). Estas prácticas pueden conducir a un nuevo enfoque de conservación y aprovechamiento sostenible, constituyendo una respuesta ante el estado de vulnerabilidad de muchas especies en su medio natural (Ruiz et al., 2004; Andrade et al., 2012). Al respecto se presentan los resultados de la inoculación in situ de Morchella spp. y su potencial como práctica en la micosilvicultura.

\section{OBJETIVOS}

Evaluar el crecimiento de cinco cepas de dos especies de Morchella in vitro y su inoculación in situ en un bosque mesófilo de montaña ubicado en el municipio de Xalapa Veracruz, México. 


\section{MATERIALES Y MÉTODOS}

Se realizó un experimento donde se evaluó el crecimiento micelial de dos especies de Morchella, en cuatro tipos de grano (maíz, avena, trigo y centeno), para determinar cuál es el más apropiado para la elaboración del agente inoculante (Alvarado et al., 2011), donde el testigo fue el trigo, ya que es el que usa comúnmente. Se utilizó un diseño experimental completamente al azar con 10 repeticiones por tratamiento. Los diversos granos se remojaron hasta obtener un porcentaje de humedad de $60 \%$ y posteriormente fueron esterilizados durante 30 minutos a $120{ }^{\circ} \mathrm{C} \mathrm{y}$ $103 \mathrm{kPa}$ (15 psi) de presión.

Las cepas utilizadas fueron: M. esculenta (IE-750) y M. conica (IE-815, IE-817, IE-816 y IE-814). La primera se importó de los Estados Unidos y las otras cuatro fueron colectas en el Estado de México en un bosque de pino (LN $100^{\circ} 01^{\prime} 46.30 ”$, LW $\left.19^{\circ} 18^{\prime} 08.73^{\prime \prime}\right)$. Estas fueron identificadas a través de un análisis de amplificación de genes de la región ITS (Internal Transcript Spacer) del ADN ribosomal (Kellner et al., 2007) y se encuentran resguardadas en el Cepario del Instituto de Ecología, A.C. (Inecol) bajo condiciones de crioconservación. Cada una de las cepas se sembró en medio de cultivo con extracto de composta $(20$ g de malta +20 g de agar $+800 \mathrm{ml}$ de extracto de composta para champiñón $1^{-1}$ ) (Savoie et al., 2001), esterilizado bajo condiciones convencionales y se incubaron a $26{ }^{\circ} \mathrm{C}$ en oscuridad para la obtención del micelio.

Para determinar el crecimiento micelial se introdujeron $10 \mathrm{~g}$ de cada grano por caja Petri y se colocó un implante circular de $0.5 \mathrm{~mm}$ de diámetro por cada cepa, incubando a $27^{\circ} \mathrm{C}$ en condiciones de oscuridad, durante nueve días. Este se determinó en centímetros cuadrados trazando la superficie de crecimiento cada tercer día para cada réplica. La superficie de crecimiento se determinó con el apoyo del programa Arc View GIS 3.12.

A partir de los resultados obtenidos se realizó un cuasiexperimento, en donde se inocularon $100 \mathrm{~g}$ del tratamiento con mayor crecimiento micelial, en cinco sitios bajo sombra completa, ubicados de manera radial en los remanentes de un bosque mesófilo de montaña (LN 19³1'11.68”, LW 96 56'15.31”), con predominancia de especies del género Inga, Liquidambar y Sambucus, con una altitud de $1400 \mathrm{~m}$ snm, en un suelo clasificado como Andosol húmico (pH 6.5 y 21\% de humedad y textura franco arcillosa). Los granos inoculados se depositaron a cielo abierto y se cubrieron con un cartón corrugado de $30 \mathrm{~cm} \times 30 \mathrm{~cm}$ a manera de acolchado, para impedir una evaporación excesiva y evitar la presencia de malezas, se regaron con agua corriente, cada tres días por dos semanas. La inoculación se realizó el 26 de marzo del 2009.

\section{RESULTADOS}

En el experimento, el crecimiento micelial en los diferentes tipos de grano mostró diferencias significativas entre tratamientos (Tukey, $\mathrm{P}<0.05$ ), siendo el centeno (28.6 $\mathrm{cm}^{2}$ ) el de mayor crecimiento de todas las cepas analizadas, seguido de la avena $\left(15.2 \mathrm{~cm}^{2}\right)$, trigo $\left(6.5 \mathrm{~cm}^{2}\right)$ y maíz $\left(4.5 \mathrm{~cm}^{2}\right)$ (Fig. 1). Asimismo, la cepa con mayor respuesta entre tratamientos (Tukey, $\mathrm{P}<0.05$ ) fue la IE-817 (17.9 $\mathrm{cm}^{2}$ ) con respecto a las demás (IE-815=16.4 $\mathrm{cm}^{2}$; IE- $816=14.9 \mathrm{~cm}^{2}$; IE- $814=10.7 \mathrm{~cm}^{2}$ y IE- $\left.750=8.4 \mathrm{~cm}^{2}\right)$. Por ello, se eligió el grano de centeno y la cepa IE-817 ( $M$. conica) para la siguiente fase.

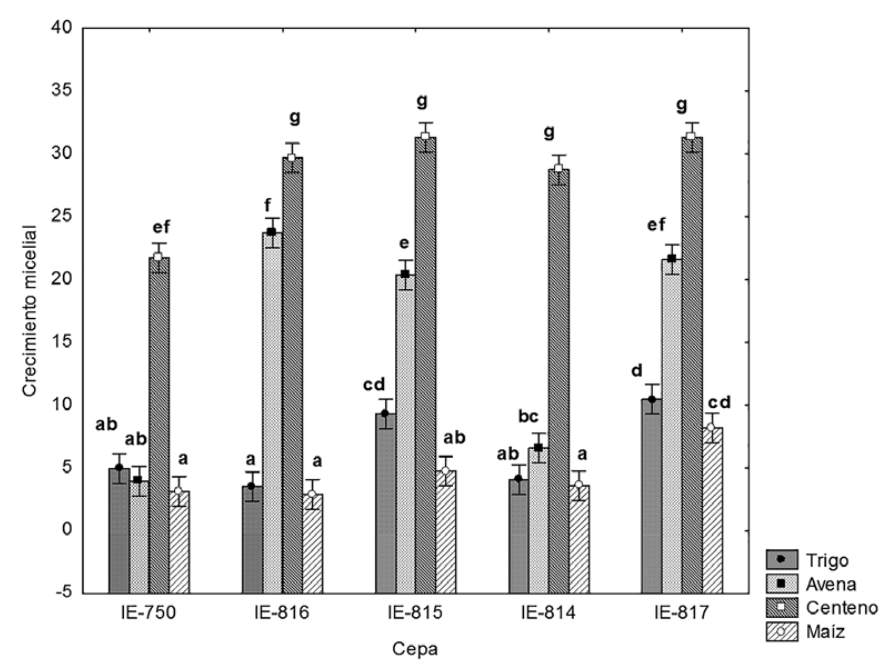

FIgURA 1. Crecimiento micelial de las cinco cepas estudiadas en diferentes tipos de grano.

Tratamientos con diferente letra son estadísticamente diferentes (Tukey, P<0.05). 
Del cuasiexperimento se obtuvieron tres periodos de fructificación, el primero fue a casi dos meses después de la inoculación (4 de junio de 2009) y generó un esporocarpo de $75 \mathrm{~mm}$ de altura, el segundo (24 de julio de 2011) produjo tres individuos de $63 \mathrm{~mm}, 40 \mathrm{~mm}$ y $25 \mathrm{~mm}$ respectivamente y el tercero (7 de septiembre de 2011) dio lugar a cinco ejemplares $(43,31,21,12$, y 8$) \mathrm{mm}$ (Fig. 2). Ninguno de ellos asociado (visiblemente) a los arboles aledaños. Se puede observar que conforme avanzó el tiempo los especímenes disminuyeron de tamaño pero incrementaron su número.

Todos los ejemplares se recolectaron en su estado de madurez y se encuentran resguardados en la colección de hongos del Herbario del Instituto de Ecología A.C., en la colecta $\mathrm{S} / \mathrm{N}$, con las fechas indicadas anteriormente.

\section{DISCUSIÓN}

Una de las principales limitantes en la producción controlada de Morchella spp. es el desconocimiento de su ciclo de vida y de sus interacciones ecológicas, ya que acorde con las condiciones ambientales, puede comportarse como un hongo saprofito, micorrízico o facultativo (Stamets, 1993), lo que dificulta su entendimiento y limita aún más su producción bajo condiciones controladas. Por lo que la estrategia más adecuada para su producción, es su propagación a partir de un esquema de micosilvicultura.

$\mathrm{Al}$ respecto, diversos estudios han demostrado que es posible obtener hongos en condiciones naturales, a través de su inoculación directa en campo o con el uso de plantas micorrizadas (Kües y Liu, 2000; Morcillo y Sánchez, 2004; Jiménez et al., 2013), aunque no existen estudios con respecto a Morchella spp, destacan los trabajos realizados en diferentes especies de trufas (Tuber melanosporum Vittad, T. uncinatum Chatin, T. borchii Vittad) (Morcillo y Sánchez, 2004) y algunos avances en la producción de Tricholoma magnivelare (Peck) Redhead, T. matsutake (S. Ito \& S. Imai) Singer (Iwase, 1997) y Cantharellus cibarius Fr (Danell y Camacho, 1997).

En México se han utilizado asociaciones de hongos con especies forestales, tales como Amanita caesarea (Scop) Pers con Pinus patula Schl. et Cham y Laccaria laccata (Scop) Cooke con P. greegii Englem, P. patula y $P$. pseudostrobus Lindl (Jiménez et al., 2013). Adicionalmente los hongos sirven de alimento a la fauna local, la cual a su vez contribuye a su dispersión y a la regeneración vegetal en zonas perturbadas ya que las excretas son excelente fuente de inóculo micorrizógeno (Castillo-Guevara et al., 2012), de tal manera que los hongos son vitales en

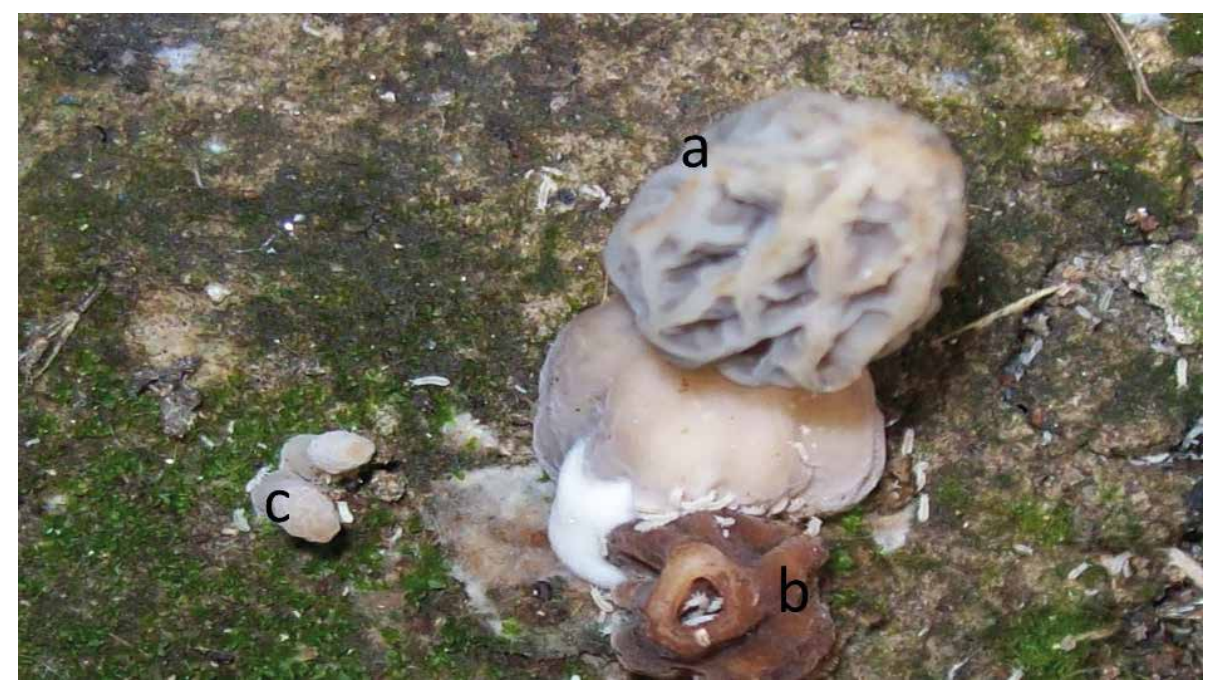

Figura 2. Ejemplares de Morchella conica obtenidos in situ, durante el segundo periodo de fructificación: a=ejemplar en estado de madurez, b=ejemplar dañado y en estado de descomposición, c=primordios de nuevos ejemplares en crecimiento. 
el funcionamiento del ecosistema. Esto, junto con el conocimiento tradicional existente, puede orientar el manejo integral del bosque (Savoie y Largeteau, 2011).

A pesar de que no se pueden correlacionar los resultados con condiciones ambientales, cantidad de inóculo, relación con especies arbóreas, etc., los hallazgos indican que la inoculación in situ puede dar lugar a procesos de domesticación y cultivo de hongos silvestres, (especialmente micorrícicos) a escala comercial (Kües y Liu, 2000), siempre y cuando se considere al bosque como una parte integral de este proceso. En este sentido, la importancia de este trabajo reside en la posibilidad de contribuir con un nuevo enfoque a la producción de este hongo a través de su uso como inóculo y bioinoculante para micorrización artificial (Savoie y Largeteau, 2011), tratando de simular sus condiciones de reproducción, tal como se realizó para los primeros hongos domesticados (Chang y Miles, 2004). Esta estrategia de producción in situ, no solo favorecería la obtención de Morchella spp, si no que puede contribuir a la salud y estabilidad forestal, a través de funciones ecológicas (simbiosis, descomposición de residuos lignocelulósicos, mantenimiento de la fertilidad del suelo, etc.), económicas (generación de ingresos por recolección) y sociales (manejo del bosque bajo modelos silvícolas).

Adicionalmente, este tipo de estrategia podría representar una importante forma de conservación del recurso e impulsaría un proceso de domesticación con un enfoque que guarde el equilibrio entre aprovechamiento y conservación (Garibay-Orijel et al., 2006), ya que en México el consumo de hongos forma parte del acervo cultural y estrategias de subsistencia de la población rural que vive en las zonas boscosas del país (Alvarado-Castillo y Benítez, 2009). Esta alternativa productiva (aunque preliminar y sujeta a nuevos estudios) podría ser competitiva en un esquema de mercado y constituir una respuesta ante el estado de vulnerabilidad por sobreexplotación de esta y otras especies de hongos comestibles en su medio natural (Ruiz et al., 2004).

Aunque este trabajo tiene el carácter de preliminar y solo muestra el potencial de la inoculación in situ, aún faltan más estudios, que consideren las características locales (altitud, pendiente, orientación) estructura del bosque (densidad, edad, especies presentes) y variables climáticas (precipitación y temperatura) (Savoie y Largeteau 2011), además de la recopilación y sistematización del conocimiento tradicional, tanto en esta como en otras especies de hongos para constituir una verdadera estrategia enfocada a un esquema complejo de micosilvicultura.

\section{CONCLUSIONES}

El hallazgo vislumbra a la inoculación in situ como una alternativa factible para la producción de Morchella (particularmente de M. conica), por medio de la utilización de centeno como agente de dispersión. No obstante se requieren de mayor cantidad de estudios en aspectos propios de la micosilvicultura, por ejemplo, a través de la generación de modelos para la identificación de zonas potenciales para su reproducción, su uso como bioinoculante, asociación con la masa forestal, etc. De ser así el manejo de este hongo podría contribuir a las economías locales y ser un factor de transformación social de enorme relevancia en los ecosistemas forestales.

\section{AGRADECIMIENTOS}

Los autores agradecen al Instituto de Ecología, A.C. por las facilidades otorgadas para realizar esta investigación y al Consejo Nacional de Ciencia y Tecnología (Conacyt) por el financiamiento a través de la beca de Doctorado No 175325. Así como a los revisores anónimos por las contribuciones al manuscrito.

\section{REFERENCIAS}

Alvarado-Castillo G. y G. Benítez. 2009. El enfoque de agroecosistemas como una forma de intervención científica en la recolección de hongos silvestres comestibles. Tropical and Subtropical Agroecosystems 10:531-539.

Alvarado-Castillo, G., G. Mata, A. Pérez-Vázquez, D. Martínez-Carrera, M.E. Nava-Tablada, F. Gallardo-López y F. Osorio-Acosta. 2011. Morchella sclerotia production through grain supplementation. Interciencia 36:768-773.

Andrade G.R.H., G. Mata y J.E. Sánchez. 2012. La producción iberoamericana de hongos comestibles en el contexto 
internacional. In: J.E. Sánchez y G. Mata, eds. Hongos comestibles y medicinales en Iberoamérica: investigación y desarrollo en un entorno multicultural. El Colegio de la Frontera Sur - Instituto de Ecología, A.C., Tapachula, Chiapas. p:9-18.

Barnes, S. y A. Wilson. 1998. Cropping of the french black morel a preliminary investigation. Rural Industries Research and Development Corporation. Australia. 14 p.

Bonet, J.A., M. Palahí, C. Colinas, T. Pukkala, C.R. Fischer, J. Miina y J. Martínez de Aragón. 2010. Modeling the production and species richness of wild mushrooms in pine forests of the Central Pyrenees in northeastern Spain. Canadian Journal of Forest Research 40(2):347-356.

Bonet, J.A., S. De-Miguel, J. Martínez de Aragón, T. Pukala y M. Palahí. 2012. Immediate effect of thinning on the yield of Lactarius group deliciosus in Pinus pinaster forests in Northeastern Spain. Forest Ecology and Management 265:211-217.

Castillo-Guevara, C., C. Lara y G. Pérez. 2012. Micofagia por roedores en un bosque templado del centro de México. Revista Mexicana de Biodiversidad 83:772-777.

Chang, S.T. y P.G. Miles. 2004. Mushroom cultivation, nutritional value, medicinal effect, and environmental impact. CRC Press. Washington. 480 p.

Danell, E. y F.J. Camacho. 1997. Successful cultivation of the golden chanterelle. Nature 385:303.

Garibay-Orijel, R., J. Cifuentes, A. Estrada-Torres y J. Caballero. 2006. People using macro-fungal diversity in Oaxaca, Mexico. Fungal Diversity 21:41-67.

Iwase, K. 1997. Cultivation of mycorrhizal mushroom. Food Reviews International 13:431-442.

Jiménez, R.M., J. Pérez-Moreno, J.J. Almaraz-Suárez y M. Torres-Aquino. 2013. Hongos silvestres con potencial nutricional, medicinal y biotecnológico comercializados en Valles Centrales, Oaxaca. Revista Mexicana de Ciencias Agrícolas 4(2):199-213.

Kellner, H., P. Luis y F. Buscot. 2007. Diversity of laccase-like multicopper oxidase genes in Morchellaceae: identification of genes potentially involved in extracellular activities related to plant litter decay. FEMS Microbiology Ecology 61:153-163.
Kües, U. y Y. Liu. 2000. Fruiting body production in basidiomycetes. Applied Microbiology Biotechnology 54:414-152.

Martínez de Aragón, J., P. Riera, M. Giergiczny y C. Colinas. 2011. Value of wild mushroom picking as an environmental service. Forest, Policy and Management 252(1-3):239256.

Martínez-Peña, F., S. de-Miguel, T. Pukkala, J.A. Bonet, P. Ortega-Martínez, J. Aldea y J. Martínez de Aragón. 2012. Yield models for ectomycorrhizal mushrooms in Pinus sylvestris forests with special focus on Boletus edulis and Lactarius group deliciosus. Forest Ecology and Management 282:63-69.

Masaphy, S. 2005. External ultrastructure of fruit body initiation in Morchella. Mycological Research 109:508-512.

Molina, R., T. O’Dell, D. Luoma, M. Amaranthus, M. Castellano y K. Russell. 1993. Biology, ecology, and social aspects of wild edible mushrooms in the forests of the Pacific Northwest: a preface to managing commercial harvest. Department of Agriculture, Forest Service, Pacific Northwest Research Station. Portland. 42 p.

Montoya, S. y C.E. Orrego. 2012. Growth, fruiting and lignocellulolytic enzyme production by the edible mushroom Grifola frondosa (maitake). World Journal of Microbiology and Biotechnology 28(4):1533-1541.

Morcillo, M. y M. Sánchez. 2004. ¿Por qué es tan difícil cultivar hongos micorrícicos comestibles?. Terralia 45:80-85.

Ower, R.D. 1982. Notes on the development of the morel ascocarp: Morchella esculenta. Mycologia 74:142-144.

Ower, R.D., G.L. Mills y J.A. Malachowski. 1986. Cultivation of Morchella. U.S. Patent No: 4.594.809.

Ower, R.D., G.L. Mills y J.A. Malachowski. 1988. Cultivation of Morchella. U.S. Patent No 4.757.640.

Pilz, D., R. McLain, S. Alexander, L. Villarreal-Ruiz, S. Berch, T. Wurtz, C. Parks, E. McFarlane, B. Baker, R. Molina y J.E. Smith. 2007. Ecology and management of morels harvested from the forests of western North America. Department of Agriculture, Forest Service, Pacific Northwest Research Station. Portland. 161 p.

Ruiz, P.M., B. Belcher, R. Achdiawan, M. Alexaides, C. Aubertin, J. Caballero, B. Campbell, C. Clement, T. Cun- 
ningham, A. Fantini, H. de Foresta, C. García-Fernández, K.H. Gautam, P. Hersch-Martínez, W. de Jong, K. Kusters, M.G. Kutty, C. López, M. Fu, M.A. MartínezAlfaro, T.R. Fair, O. Ndoye, R. Ocampo, N. Rai, M. Ricker, K. Schereckenberg, S. Shackleton, P. Shanley, T. Sunderland y Y. Youn. 2004. Markets drive the specialization strategies of forest peoples. Ecology and Society 9:1-29.

Savoie, J.M. y M.L. Largeteau. 2011. Production of edible mushrooms in forests: trends in development of a mycosilviculture. Applied Microbiology and Biotechnology 89:971-979.

Savoie, J.M., R. Iapicco y M.L. Largeteau. 2001. Factors influencing the competitive saprophytic ability of Trichoderma harzianum Th2 in mushroom (Agaricus bisporus) compost. Mycological Research 105(II):1348-1356.

Semarnat (Secretaria de Medio Ambiente y Recursos Naturales). 2010. Norma Oficial Mexicana NOM-059-Semarnat-2010, Protección ambiental-Especies nativas de
México de flora y fauna silvestres-Categorías de riesgo y especificaciones para su inclusión, exclusión o cambioLista de especies en riesgo. Diario Oficial de la Federación. México. 2010(1):1-30.

Stamets, P. 1993. Growing gourmet and medicinal mushroom. Ten Speed Press, Berkeley. 614 p.

Stott, K. y C. Mohammed. 2004. Specialty mushroom production systems: maitake and morels. Rural Industries Research and Development Corporation, Australia. 78 p. Tacón, A. y J. Palma. 2006. La comercialización de los productos forestales no madereros: una oportunidad para el manejo comunitario y la valoración del bosque nativo. Revista Bosque Nativo 39(2):253-266.

Manuscrito recibido el 22 de abril de 2014. Aceptado el 21 de septiembre de 2015

Este documento se debe citar como:

Alvarado-Castillo, G., G. Benítez-Badillo y G. Mata. 2015. Inoculación in situ de Morchella: primer registro de su obtención en México. Madera y Bosques 21(3):129-135. 